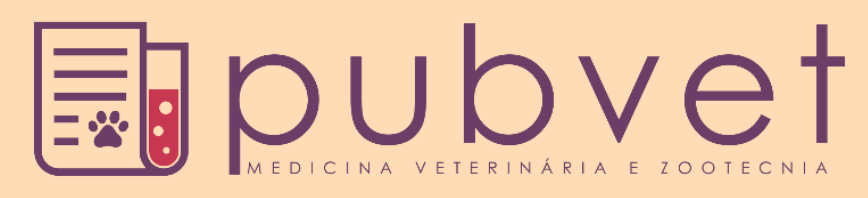

https://doi.org/10.31533/pubvet.v15n05a803.1-4

\title{
Ocorrência de seroma em cavidade anoftálmica em Bugio-ruivo (Alouatta guariba clamitans)
}

\author{
Bruna Zafalon da Silva $^{1 *}$, Rochelle Gorczak ${ }^{1}$, Thanize Lopes ${ }^{(2)}$, Camila Schmitz $\mathbb{1}^{2}$, Victoria \\ Regina Schmidt $\mathbb{1}^{3}$, Marilia Avila Valandro ${ }^{1}$, Flávio Rybu $\mathbb{1}^{4}$ \\ ${ }^{I}$ Professora do Centro Universitário Ritter dos Reis - UniRitter, Faculdade de Medicina Veterinária, Porto Alegre-RS, Brasil. \\ ${ }^{2}$ Acadêmica do Centro Universitário Ritter dos Reis - UniRitter, Faculdade de Medicina Veterinária, Porto Alegre-RS, Brasil. \\ ${ }^{3}$ Acadêmica da Universidade Federal do Rio Grande do Sul, Curso de Medicina Veterinária, Porto Alegre-RS, Brasil. \\ ${ }^{4}$ Médico Veterinário Clínica Veterinária Cristal, Porto Alegre-RS, Brasil. \\ *Autor para correspondência, E-mail: brunazs@gmail.com
}

Resumo. O seroma é classificado como acumulo de fluido tecidual extracelulares, no espaço morto, entre os planos teciduais de uma ferida cirúrgica, podendo gerar um aumento de volume progressivo no local. O objetivo do presente relato é descrever a ocorrência de seroma em cavidade anoftálmica de bugio-ruivo (Alouatta guariba clamitans). Foi atendido com aumento de volume ocular, um bugio-ruivo, fêmea, infante (aproximadamente um ano de idade), após 6 meses de ter realizado o procedimento de enucleação com blefarorrafia. Ao exame físico, realizado mediante contenção química do paciente (cetamina $8 \mathrm{mg} / \mathrm{kg}$, IM), o animal não apresentou alterações sistêmicas dignas de nota, e na avaliação oftálmica foi constatado somente o aumento de volume de cavidade anoftálmica, de consistência mole. Para fins diagnósticos, foi realizada punção, mediante uso de agulha hipodérmica $22 \mathrm{G}$ e seringa estéril, sendo coletado $4,5 \mathrm{ml}$ de liquido translucido presente em cavidade anoftálmica. Em analise citológica do material, foi observada a presença de 2 leucócitos por campo analisado e $2 \mathrm{~g} / \mathrm{dL}$ de proteína. A partir disto, foi diagnosticado a presença de seroma. Conclui-se que o tratamento paliativo de drenagem ocular se mostrou efetivo para o paciente, sendo recomendada drenagem periódica.

Palavras chave: complicações cirúrgicas, oftalmologia, olhos, primatas

\section{Occurrence of seroma in anolphtalmic cavity in Alouatta guariba clamitans}

\begin{abstract}
The seroma is classified as an accumulation of extracellular tissue fluid, in the dead space, between the tissue planes of a surgical wound, which can generate a progressive increase in volume at the site. The purpose of this report is to describe the occurrence of seroma in anophthalmic cavity of red howler (Alouatta guariba clamitans). A red-howler, female, infant (approximately 1 year old) was attended with an increase in ocular volume, after 6 months of having performed the enucleation procedure with blepharorraphy. Upon physical examination, carried out by means of chemical restraint of the patient (ketamine $8 \mathrm{mg} / \mathrm{kg}$, IM), the animal did not present any systemic changes worthy of note, and in the ophthalmic evaluation, only an increase in the volume of anophthalmic cavity, of soft consistency, was found. For diagnostic purposes, a puncture was performed using a $22 \mathrm{G}$ hypodermic needle and sterile syringe, and $4.5 \mathrm{ml}$ of translucent liquid present in anophthalmic cavity was collected. In cytological analysis of the material, the presence of 2 leukocytes per analyzed field and $2 \mathrm{~g} / \mathrm{dL}$ of protein was observed. From this, the presence of seroma was diagnosed. It is concluded that palliative treatment of ocular drainage was effective for the patient, periodic drainage being recommended.
\end{abstract}

Keywords: Surgical complications, ophthalmology, eyes, primates 


\section{Aparición de seroma en cavidad anoftálmica de Alouatta guariba clamitans}

Resumen. El seroma se clasifica como una acumulación de líquido tisular extracelular, en el espacio muerto, entre los planos tisulares de una herida quirúrgica, que puede generar un aumento progresivo de volumen en el sitio. El propósito de este informe es describir la aparición de seroma en la cavidad anoftalmica de los monos aulladores (Alouatta guariba clamitans). Una hembra, joven (aproximadamente 1 año de edad) que fue atendida con un aumento en el volumen ocular, 6 meses después del procedimiento de enucleación con blefarrorrafía. En el examen físico, realizado mediante contención química del paciente (ketamina $8 \mathrm{mg} / \mathrm{kg}$, IM), el animal no presentó cambios notables, con solo un aumento en el volumen de la cavidad anoftalmica, de consistencia blanda. Para fines de diagnóstico, se realizó una punción con una aguja hipodérmica $22 \mathrm{G}$ y una jeringa estéril, con 4,5 $\mathrm{ml}$ de líquido translúcido presente en la cavidad anoftalmica recogida. En el análisis citológico del material, se observó la presencia de 2 leucocitos por campo analizado y $2 \mathrm{~g} / \mathrm{dL}$ de proteína. A partir de esto, se diagnosticó la presencia de seroma. Se concluye que el tratamiento paliativo del drenaje ocular fue efectivo para el paciente, por lo que se recomienda el drenaje periódico.

Palabras clave: complicaciones quirúrgicas, oftalmología, ojos, primates, seroma

\section{Introdução}

O bugio-ruivo (Alouatta guariba clamitans) é uma espécie suscetível ao impacto antrópico, principalmente, por degradação de habitats, sendo considerada vulnerável (Buss et al., 2015; Machado et al., 2008). Como consequência deste impacto, muitos indivíduos resgatados que são reabilitados podem apresentar inaptidão à reintrodução em vida livre, conforme critérios pré-estabelecidos pela legislação vigente, o que leva a destinação destes animais ao cativeiro devido as sequelas ou comportamento estereotipados (Zafalon-Silva et al., 2019).

$\mathrm{Na}$ rotina de atendimento clínico de primatas, traumas oculares podem ser observados (Galera et al., 2002), o que leva em a indicação de enucleação quando há perfurações oculares, endoftalmite, panoftalmite, ruptura do nervo óptico, neoplasias intraoculares, traumatismos severos (Bernhard \& Simon, 2003; Bojrab, 2005; Rahal et al., 2000).

A enucleação consiste em um procedimento cirúrgico de rotina, onde é realiza a remoção do globo ocular como um todo, incluindo o revestimento fibroso interno (Bernhard \& Simon, 2003; Bojrab, 2005; Goes et al., 2012; Rahal et al., 2000). Como complicações podem ser citadas coleções líquidas, também conhecidas como seromas. Estas são patologias relativamente comuns no pós-operatórios de rotina de cirurgias reconstrutivas que consistem no acumulo de fluidos teciduais extracelulares, dentro do espaço morto, entre os planos teciduais de uma ferida cirúrgica, podendo gerar um aumento de volume progressivo no local, de forma aguda ou crônica. São considerados benigno e não acarretando em maiores complicações para o indivíduo (Janis, 2016; Scheffer et al., 2013; Slatter, 2007).

Partindo deste contex to, o objetivo do presente relato é descrever a ocorrência de seroma em cavidade anoftálmica de bugio-ruivo (Alouatta guariba clamitans).

\section{Relato do caso}

Um bugio-ruivo (Alouatta guariba clamitans), 1 ano, fêmea foi atendido com aumento de volume ocular, 6 meses após o procedimento de enucleação com blefarorrafia devido a trauma em nervo óptico. Ao exame físico, realizado mediante a contenção química (cetamina $8 \mathrm{mg} / \mathrm{kg}$, IM), o animal foi avaliado fisicamente onde não foram observadas alterações sistêmicas dignas de nota. Após, foi realizado exame oftálmico, onde foi constatado aumento de volume em toda região de cavidade anoftálmica, com fragilidade cutânea, aumento de volume considerável, com consistência mole, compatível com uma coleção líquida (Figura 1).

Para fins diagnósticos foi realizada antissepsia cutânea com álcool $70 \%$ e punção do aumento de volume local, mediante uso de agulha hipodérmica $22 \mathrm{G}$ e seringa, sendo coletados $4,5 \mathrm{ml}$ de líquido 
translucido presente em cavidade anofltámica (Figura 2) e encaminhado para análise citológica em frasco contendo EDTA K2.

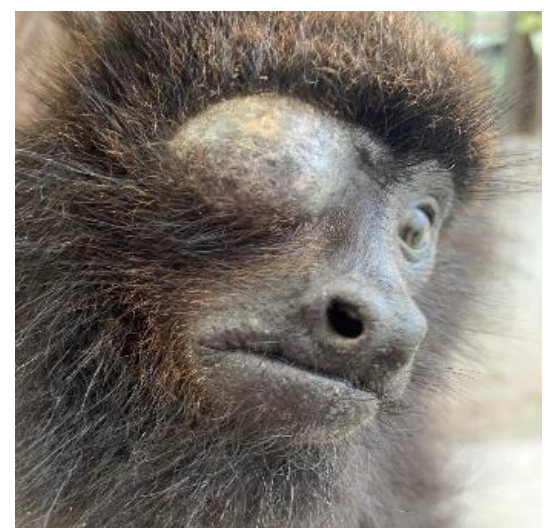

Figura 1. Aumento de volume em região de cavidade anoftálmica, com fragilidade cutânea de consistência mole em espécime de Bugioruivo (Alouatta guariba clamitans).

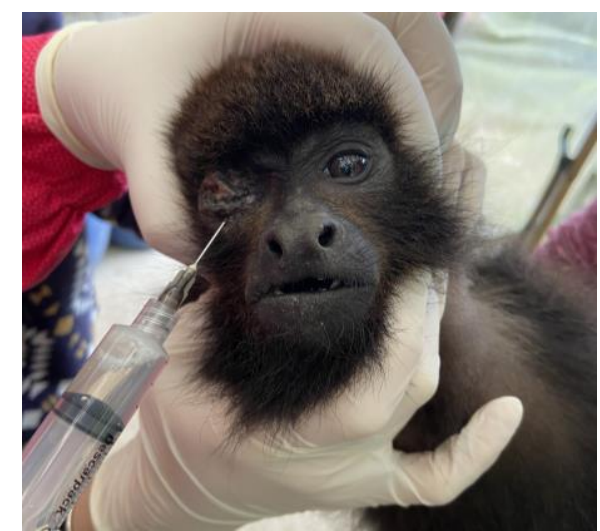

Figura 2. Realização de punção em cavidade anoftálmica de bugio-ruivo (Alouatta guariba clamitans). Presença de conteúdo translúcido em cavidade anoftálmica.

$\mathrm{Na}$ análise citológica do material coletado, foi observada baixa celularidade, sendo observada a presença de 2 leucócitos por campo analisado e $2 \mathrm{~g} / \mathrm{dL}$ de proteína. A partir disto, foi diagnosticado a presença de seroma de aparecimento tardio em um espécime de bugio-ruivo (Alouatta guariba clamitans). Desde então é realizada uma vez ao mês, ao ocorrer recidivas, a punção e drenagem da área, pois não é plausível de realização de compressão local.

\section{Discussão}

O seroma é uma das complicações pós-operatórias mais comuns na rotina cirúrgica, podendo ocorrer após enculeações onde não são utilizados enxertos ou implantes na região enucleada, como no caso acima descrito. O mesmo pode ocorrer de forma aguda no pós-operatório imediato ou de forma tardia em decorrência de reações inflamatórias crônicas, levando ao acumulo de líquidos progressivo no local (Bernhard \& Simon, 2003), como no caso descrito com aparecimento tardio de 6 meses após procedimento.

No caso clínico descrito, corrobora-se que o espaço morto deixado pela enucleação, levou a ocorrência do seroma em cavidade anoftalmica. Segundo Williams \& Moores (2003) a formação de espaço morto excessivo durante a cirurgia, ou falha em reduzi-lo adequadamente, principalmente quando associada ao resultado de dissecção excessiva ou remoção de uma grande área de tecido, podem levar a formação de seroma.

Por se tratar de um animal silvestre e levando em consideração o grau de estresse do paciente, gerado por intervenções, é de suma importância que o tratamento seja adequado para minimizar os efeitos nocivos do estresse no organismo, que pode levar a morte do animal (Miller \& Fowler, 1978). Portanto para estabelecer um tratamento, é indispensável o exame diagnóstico confirmatório, conforme o realizado no caso, através de analise citológica do líquido presente em cavidade anofitalmica, sendo diagnosticado um seroma. Visando um tratamento paliativo, e não curativo, a drenagem periódica do liquido formado, é o método mais rápido e efetivo de trazer alívio local para o paciente (Seretis et al., 2017; Williams \& Moores, 2003).

O tratamento de seromas é estritamente dependente do tamanho da formação da coleção líquida. Seromas pequenos, normalmente são autoresolutivos, não tendo muitas consequências graves de sua ocorrência. No entanto, seromas de tamanho grande possuem a indicação de drenagem, e raramente uma única aspiração resulta em resolução do problema, sendo recomendado o tratamento de drenagem periódicas. Estas aspirações devem ser realizadas usando técnicas estéreis para evitar infecções iatrogênicas da ferida, como realizado no caso descrito (Kuroi et al., 2006; Seretis et al., 2017; Shaver et al., 2014; Williams \& Moores, 2003). 


\section{Conclusão}

O tratamento paliativo de drenagem ocular mostrou-se efetivo para o bem-estar do paciente, sendo recomendada drenagem periódica, até a remissão dos sinais, podendo essa não ocorrer.

\section{Referências}

Bernhard, M. S., \& Simon, A. P. (2003). Diseases and surgery of the canine orbit. In K. N. Gelatt (Ed.), Manual de oftalmologia veterinária. Manole Ltda.

Bojrab, M. J. (2005). Técnicas atuais em cirurgia de pequenos animais. Editora Roca.

Buss, G., Romanowski, H. P., \& Becker, F. G. (2015). O bugio que habita a mata e a mente dos moradores de Itapuã-Uma análise de percepção ambiental no entorno do Parque Estadual de Itapuã, Viamão, RS. Revista Biociências, 21(2), 14-28.

Galera, P. D., Ávila, M. O., Ribeiro, C. R., \& Santos, F. V. (2002). Estudo da microbiota da conjuntiva ocular de macacos-prego (Cebus apella-LINNAEUS, 1758) e macacos bugio (Alouatta carayaHUMBOLDT, 1812), provenientes do reservatório de Manso, MT, Brasil. Arquivo Do Instituto de Biologia, 69(2), 33-36.

Goes, L. D., Risseti, R. M., Dias, F. G. G., Pereira, D. M., \& Dias, L. G. G. G. (2012). Técnica cirúrgica de enucleação-Revisão de Literatura. Revista Científica Eletrônica de Medicina Veterinária-ISSN, $18,1679-7353$.

Janis, I. L. (2016). Psychological stress: Psychoanalytic and behavioral studies of surgical patients. American Sociological Review, 24(3). https://doi.org/10.2307/2089417.

Kuroi, K., Shimozuma, K., Taguchi, T., Imai, H., Yamashiro, H., Ohsumi, S., \& Saito, S. (2006). Evidence-based risk factors for seroma formation in breast surgery. Japanese Journal of Clinical Oncology, 36(4), 197-206. https://doi.org/10.1093/jjco/hyl019.

Machado, A. B. M., Drummond, G. M., \& Paglia, A. P. (2008). Livro vermelho da fauna brasileira ameaçada de extinção. In Livro vermelho da fauna brasileira ameaçada de extinção (p. 1420).

Miller, R. E., \& Fowler, M. E. (1978). Fowler's Zoo and Wild Animal Medicine Current Therapy.

Rahal, S. C., Bergamo, F. M. M., \& Ishiy, H. M. (2000). Prótese intra-ocular de resina acrílica em cães e gatos. Arquivo Brasileiro de Medicina Veterinária e Zootecnia, 52(4), 319-324. https://doi.org/10.1590/s0102-09352000000400005.

Scheffer, J. P., Atallah, F. A., Gomes, C., Estupñan, O. F. T., Silva, S. J. Q., Silva, T. I. R., Vale, D. F., \& Oliveira, A. L. A. (2013). Cirurgia reconstrutiva no tratamento de feridas traumáticas em pequenos animais. Brazilian Journal of Veterinary Medicine, 35(Supl. 1), 70-78.

Seretis, K., Goulis, D., Demiri, E. C., \& Lykoudis, E. G. (2017). Prevention of seroma formation following abdominoplasty: a systematic review and meta-analysis. Aesthetic Surgery Journal, 37(3), 316-323. https://doi.org/10.1093/asj/sjw192.

Shaver, S. L., Hunt, G. B., \& Kidd, S. W. (2014). Evaluation of fluid production and seroma formation after placement of closed suction drains in clean subcutaneous surgical wounds of dogs: 77 cases (2005-2012). Journal of the American Veterinary Medical Association, 245(2), 211-215. https://doi.org/10.2460/javma.245.2.211.

Slatter, D. H. (2007). Manual de cirurgia de pequenos animais (Vol. 2). Manole São Paulo.

Williams, J., \& Moores, A. (2003). Drenos cirúrgicos no tratamento da ferida e na cirurgia reconstrutiva. In J. Williams \& A. Moore (Eds.), Manual de Feridas de Cães e Gatos. Roca, Brasil.

Zafalon-Silva, B., Soares, F. A. C., Pavarini, S. P., Goulart, M. de A., Snel, G. G. M., González, F. H. D., Vaz, D. B., Lopes, B. C., Oliveira, M. A. S., \& Alievi, M. M. (2019). Bacterial endocarditis and increased cardiac troponin I levels in a brown howler monkey (Alouatta guariba clamitans) with an interventricular septal defect. Journal of Medical Primatology, 48(2), 129-132. https://doi.org/10.1111/jmp.12390.

Histórico do artigo:

Recebido: 17 de novembro de 2020. Aprovado: 10 de dezembro de 2020.

Disponível online: 31 de março de 2021.
Licenciamento: Este artigo é publicado na modalidade Acesso Aberto sob a licença Creative Commons Atribuição 4.0 (CC-BY 4.0), a qual permite uso irrestrito, distribuição, reprodução em qualquer meio, desde que o autor e a fonte sejam devidamente creditados. 